\title{
Análisis costo-beneficio del control de vectores en la transmisión potencial de dengue
}

\author{
Pablo Wenceslao Orellano ${ }^{1}$ y Elena Pedroni ${ }^{1}$
}

Forma de citar Orellano PW, Pedroni E. Análisis costo-beneficio del control de vectores en la transmisión potencial de dengue. Rev Panam Salud Publica. 2008;24(2):113-9.

RESUMEN Objetivos. Evaluar el costo-beneficio de una intervención de control de mosquitos adultos con fumigación, junto con actividades de control de formas inmaduras de Aedes aegypti, para evitar la transmisión en una situación de alto riesgo.

Métodos. Se realizó un análisis de costo-beneficio desde una perspectiva social. Se comparó una intervención para el control de mosquitos adultos y formas inmaduras contra una hipótesis de no-intervención, de enero a abril de 2007, en la ciudad de Clorinda, Argentina. Se consideraron los costos directos y los beneficios indirectos e intangibles, estandarizados a dólares internacionales (I\$) utilizando la paridad de poder adquisitivo. Se asumió una incidencia de 50 casos de dengue por 1000 habitantes en la hipótesis de no-intervención.

Resultados. De enero a abril de 2007 se presentaron 176 casos de dengue en Clorinda, pero sólo cinco fueron autóctonos. El valor presente neto fue de I\$ 196879 en la estrategia de aplicación de control, mientras que en la de no aplicación se calculó en I\$ -106 724, lo que significaría un ahorro de I\$ 303602 (I\$ 6,46 por habitante) con la intervención. El análisis de sensibilidad evidenció que, con más de 1363 casos de dengue (incidencia total de 29 por 1000 habitantes) y un caso de dengue hemorrágico, la intervención es costo-benéfica.

Conclusiones. Los resultados sugieren que el control vectorial, incluyendo la fumigación para mosquitos adultos, debe evaluarse en situaciones de alto riesgo de transmisión como una alternativa costo-benéfica en países no endémicos.

Palabras clave Análisis costo-beneficio, dengue, control vectorial, Aedes, Argentina.

El dengue es la enfermedad viral transmitida por artrópodos con mayor magnitud epidemiológica y más amplio rango geográfico (1). Las infecciones de estos virus pueden resultar ina-

\footnotetext{
Ministerio de Salud de la Nación. Argentina. Dirección de Epidemiología. La correspondencia deberá enviarse a Pablo Wenceslao Orellano, Ministerio de Salud de la Nación, Dirección de Epidemiología, Av. 9 de Julio $19259^{\circ}$ Piso, Buenos Aires. Buenos Aires C1073ABA, Argentina. T: +54 11 4379-9237. Fax: +54 11 4379-9127. Correo electrónico: porellano@msal.gov.ar
}

parentes, o variar en severidad desde una enfermedad similar a la influenza leve, hasta el dengue hemorrágico y choque, que puede ser fatal si no es tratado (2). En la actualidad se informan casos de dengue en más de 30 países de las Américas; entre 2001 y 2005 se notificaron casi tres millones de casos de dengue, de los cuales 65000 correspondieron al hemorrágico y con 789 muertes (3). Las vacunas contra el dengue, si bien se encuentran en etapas de desarrollo avanzadas, debe- rán aún pasar por pruebas de eficacia en poblaciones expuestas (4). Por el momento, a menos que sean mejorados los programas de saneamiento y las acciones de control vectorial, la continua presencia de grandes poblaciones de mosquitos incrementa las probabilidades de futuras epidemias (5). Al inicio del siglo $X X$ se detectaron en Argentina casos autóctonos de dengue, con un brote en la provincia de Entre Ríos en 1916; no se diagnosticaron nuevos casos sino hasta 1997 (6). 
Desde entonces y hasta 2007 se han detectado brotes de dengue en las provincias de Salta, Jujuy, Misiones, Formosa y Corrientes, con más de 3000 casos autóctonos confirmados por laboratorio o por nexo epidemiológico, según datos de vigilancia epidemiológica del Ministerio de Salud de la Nación (MSN) no publicados.

Hasta el momento existen pocas estimaciones del impacto social y económico del dengue (7). Entre los puntos estudiados se encuentran la costo-efectividad de una posible vacuna pediátrica (8), la utilización de los años de vida perdidos ajustados por discapacidad (Dalys en inglés) (9-12) para estimar la carga de la enfermedad, o el empleo de métodos de evaluación de costo-efectividad para estimar la eficiencia de diferentes estrategias de control $(13,14)$. En conocimiento de los autores, no existen datos publicados sobre el costo-beneficio de acciones de control del dengue en situaciones de riesgo de transmisión. El objetivo del presente trabajo fue evaluar el costo-beneficio de una intervención de control de mosquitos adultos con fumigación, en conjunto con actividades de control de formas inmaduras de Aedes aegypti, para evitar que ocurra la transmisión local en una situación de alto riesgo.

\section{MATERIALES Y MÉTODOS}

\section{Sitio de estudio y antecedentes}

La ciudad de Clorinda $\left(25^{\circ} 17^{\prime} \mathrm{S}\right.$, $\left.57^{\circ} 43^{\prime} \mathrm{O}\right)$ pertenece a la provincia de Formosa y se localiza en el noreste de Argentina. Desde el punto de vista fitogeográfico corresponde a la subregión del Chaco húmedo (15), con bosques mezclados con palmares y sabanas (16). Clorinda se encuentra frente a la ciudad de Asunción, capital de la República del Paraguay, a la rivera del Río Pilcomayo, que constituye el límite internacional. La población asciende a 47000 habitantes (17), y existe un importante movimiento comercial con la cercana ciudad de Asunción. Clorinda cuenta con un sistema público provincial de salud que consiste en un hospital con internación y centros de salud distribuidos para atención ambulatoria. Las acciones de control vectorial local se realizan a través de agentes municipales, provinciales y con la colaboración de organizaciones civiles. En caso de emergencias por brotes, el gobierno provincial puede solicitar la intervención de operarios de control de vectores del MSN. En 2000 Clorinda tuvo su primer brote de dengue (serotipo 1), con 242 casos confirmados de fiebre de dengue (18); durante ese año se convirtió en la segunda localidad más afectada del país (19). Desde esa fecha y hasta 2006 no se registraron casos de dengue en la localidad. En 2002 los índices de abundancia vectoriales eran altos, con un Breteau de 87 y un índice de viviendas de $39 \%$ (20). Durante 2006 y 2007 se registró una importante epidemia de dengue en Asunción del Paraguay, que en junio de 2007 ascendió a 7335 casos de dengue y 16 de dengue hemorrágico (serotipo Den 3). $\mathrm{Al}$ inicio de 2007 se detectaron en Clorinda casos de dengue importados de Paraguay, así como otros autóctonos (21). No se detectaron casos de dengue hemorrágico ni de fallecimientos por dengue. Ante los primeros casos importados y debido al elevado riesgo de introducción del virus desde Asunción, el MSN y la provincia pusieron en marcha un operativo de control vectorial con adulticidas, mediante fu- migación desde vehículos y bloqueo con máquinas portátiles alrededor de las viviendas de los casos, en combinación con acciones de control de formas inmaduras del vector.

\section{Tipo de estudio, horizonte temporal, estrategias y análisis de sensibilidad}

Se realizó un estudio de costobeneficio desde una perspectiva social. Los costos y beneficios se tomaron en cuenta de enero a abril de 2007, período de mayor riesgo de transmisión de dengue en la localidad. Las estrategias consideradas fueron el control vectorial a través de la aplicación de insecticida para mosquitos adultos con máquinas pesadas en ciclos completos en la localidad a intervalos de siete días, y portátiles en las viviendas alrededor de los casos, sumado a las acciones de control de formas inmaduras, contra una situación hipotética de noaplicación de control vectorial. En la figura 1 se muestra el árbol de decisión usado para el estudio. Se aplicó un análisis de sensibilidad de una y dos vías con las principales variables de incidencia, costo y beneficio. El cálculo de los valores máximo y mínimo de las variables consideradas en el análisis de sensibilidad se describe en la siguiente sección. El modelo se construyó con el programa Decision Analysis by TreeAge (DATA) V. 3.0.5.

\begin{abstract}
FIGURA 1. Árbol de decisión con estrategias alternativas para el análisis de costo-beneficio en fiebre por dengue y dengue hemorrágico, ciudad de Clorinda, Argentina, enero a abril de 2007
\end{abstract}

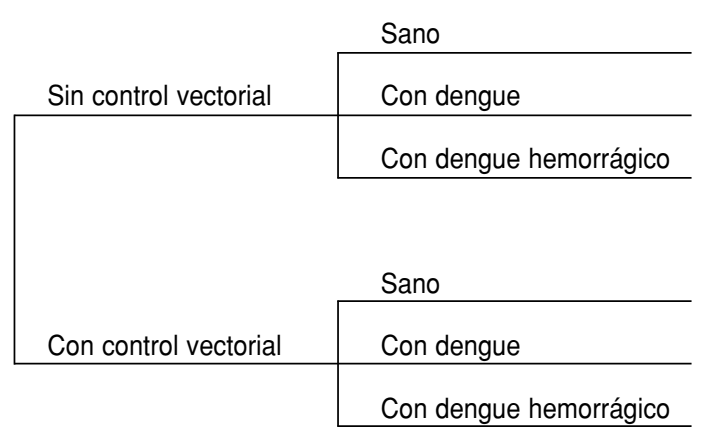

Fuente: elaboración propia. 


\section{Estimación de la incidencia}

En la estrategia de control vectorial se consideró la incidencia de casos observada, mientras que en la estrategia de no-intervención se supuso una incidencia total hipotética de 50 casos de dengue por 10000 habitantes, con un intervalo entre 0 y 100 casos por 10000 habitantes. Además se asumió una tasa dengue hemorrágico/dengue de 0,1 por 100 (línea de base), y una variación entre 0 y 1 por 100, tomando como referencia valores publicados por la Organización Panamericana de la Salud durante 2006 (22), para países con una incidencia baja de dengue hemorrágico respecto de la fiebre por dengue, como Bolivia, Brasil, Costa Rica o El Salvador.

\section{Cálculo de costos y beneficios}

Se tomaron en cuenta los costos médicos y no médicos, incluyendo el costo del personal de salud, drogas e insumos para el tratamiento del dengue y dengue hemorrágico, test de diagnóstico de laboratorio, salario y viajes de los epidemiólogos y técnicos de control vectorial, salario de los operarios locales y equipos e insumos para el control vectorial, incluyendo insecticidas y combustible para las camionetas. La información se obtuvo de entrevistas con personal administrativo y contable del MSN, responsable de la adquisición de insumos así como del pago de sueldos y viáticos (datos no publicados).

Los costos asociados a la atención médica incluyeron, para dengue clásico, el uso de paracetamol, mientras que para el dengue hemorrágico se consideró la utilización de sales de rehidratación oral, expansores plasmáticos, metoclopramida, solución fisiológica, bolsas de sangre y equipo para transfusión sanguínea, además del gasto del laboratorio de análisis clínicos. Asimismo se tomó en cuenta el costo del diagnóstico serológico confirmatorio de la enfermedad con detección de anticuerpos IgM, que se realiza en los laboratorios de la red nacional de diagnóstico. En el costo de interna- ción por dengue hemorrágico se calculó un promedio de siete días, según el valor del Nomenclador Nacional de Prestaciones Médicas de Argentina. También se consideró el salario del médico para atención de la fiebre dengue, con un promedio de dos consultas por caso.

En la estimación de los costos no médicos de la intervención se tomó en cuenta el traslado al lugar y viáticos de epidemiólogos, garantizando la presencia constante de dos profesionales con relevos cada dos semanas, durante los tres meses de mayor riesgo. De igual manera se incluyó el salario y los viáticos y traslados de ocho técnicos de control de vectores nacionales en el mismo período, para la realización de los ciclos de rociado espacial y la evaluación de las actividades de terreno. Para el nivel local municipal se calculó el salario de 30 operarios para control de formas inmaduras, durante tres meses, según datos del Ministerio de Trabajo, Empleo y Seguridad Social de Argentina (ingreso medio de ocupados plenos para el tercer trimestre del año 2006) (23). También se tomó en cuenta el trabajo de 25 operarios de organizaciones civiles, mediante el cálculo de $50 \%$ del salario antes mencionado, debido a que las tareas se realizan en jornadas reducidas.

Los insumos calculados incluyeron el insecticida organofosforado para formas inmaduras del vector, para bloqueo de casos y apoyo de las tareas de eliminación de criaderos por medios físicos. De igual forma, se estimó el insecticida piretroide para control de adultos en bloqueos de casos y en rociados - un total de 18 durante los tres meses-, completando un ciclo de rociado en toda la localidad cada cinco días. Por otra parte, se calculó el combustible para el traslado de los vehículos, movilidad interna y el movimiento de los mismos durante el rociado. Para la dilución del insecticida se utilizó agua en lugar de combustible, y no se consideró el costo. También se consideró el costo del material de difusión en forma de folletos para entrega en las viviendas durante las actividades de control; en total se imprimieron 30000 folletos.
El beneficio fue de cero en el caso de la estrategia de no intervención, mientras que para la de intervención se asumió el beneficio indirecto del ahorro de días laborales no perdidos de los 50 casos por 10000 habitantes (2 350 casos para Clorinda) y los dos casos de dengue hemorrágico que se habrían evitado. Para ello se estimó un ausentismo promedio de cinco días para la fiebre por dengue, con un rango comprendido entre 3 y 10 días en el análisis de sensibilidad. En el caso del dengue hemorrágico se consideró un ausentismo promedio de 14 días, con un rango entre 9 y 30 días. El salario utilizado fue el ingreso medio de ocupados plenos para el tercer trimestre del año 2006, según se explicó anteriormente (23).

De otro lado, se consideraron los beneficios intangibles como la "disposición a pagar", que se refiere a lo que las personas estarían dispuestas a pagar por no sufrir un episodio de la enfermedad, medido a través del método de valoración contingente (24). Para el caso del dengue se utilizaron como referencia estudios sobre cuadros similares, tales como la influenza no complicada y el resfriado común (25-27); el valor máximo fue aproximado a partir de la literatura; el mínimo fue el precio de mercado local de medicamentos para tratamiento del síndrome gripal, y la línea de base fue un promedio entre ambos valores. Para el caso del dengue hemorrágico se empleó la disposición a pagar para otra enfermedad grave como la cardiopatía isquémica (28), debido a que en la literatura no se encontraron datos relativos a la disposición a pagar para dengue hemorrágico. En ese caso se varió en +/- 50\% para obtener los valores máximo y mínimo en el análisis de sensibilidad.

Los valores monetarios de los costos y beneficios fueron estandarizados a dólares internacionales (I\$) utilizando el factor de conversión de paridad de poder adquisitivo (purchasing power parity) del año 2004, equivalente a 0,9 para Argentina (29). Cuando se utilizaron los valores de estudios realizados en otros países y presentados en dólares americanos, se realizó primero la 
conversión a la moneda original de acuerdo con la cotización del momento presentada por los autores, y luego se convirtió a dólares internacionales de acuerdo con el factor de conversión de paridad de poder adquisitivo del país. No se tomó en cuenta la tasa de descuento ni tampoco la influencia de la inflación, debido a la brevedad del tiempo considerado.

Para el análisis de costo-beneficio se usó el valor presente neto (VPN), según la siguiente ecuación:

$$
\mathrm{VPN}=\sum \text { (beneficios }- \text { costos). }
$$

\section{RESULTADOS}

Los costos directos y beneficios indirectos e intangibles calculados por ítem tanto para la estrategia de control vectorial, como para aquella de no intervención, se presentan en el cuadro 1. El costo total de la estrategia de intervención fue mayor: I\$ 378 372, es decir de I\$ 8,05 por habitante, mientras que para la estrategia de no-aplicación de control vectorial se calculó en I\$ 106724 , o I\$ 2,27 por habitante. Sin embargo, el VPN fue de I\$ 196879 en la estrategia de aplicación de control, mientras que en la de no-intervención se calculó en I\$ -106 724, lo que significaría un ahorro de I\$ 303602 o I\$ 6,46 por habitante con las medidas de control vectorial. Los valores totales de costos, beneficios, PVN y el valor marginal, se presentan en el cuadro 2 .

El análisis de sensibilidad mostró que sería necesaria una incidencia total de dengue (casos autóctonos e importados) superior a 29 casos por 1000 habitantes (1 363 casos de dengue y más de un caso de dengue hemorrágico para Clorinda) para que la estrategia de control vectorial resultara más interesante desde el punto de vista costobeneficio (figura 2), siendo en este caso el valor presente neto igual a I\$ -62 279, lo que significa un costo de I\$ 1,32 por habitante. La variación en las otras variables estudiadas como la tasa de dengue hemorrágico/dengue, el ahorro de días laborables y la disponibilidad a pagar por no sufrir un episodio de dengue o de dengue he-

CUADRO 1. Costos y beneficios directos, indirectos e intangibles, de dos estrategias, con y sin control vectorial, en presencia de dengue y dengue hemorrágico, ciudad de Clorinda, Argentina, enero a abril de 2007

\begin{tabular}{|c|c|c|c|}
\hline Variable & Estrategia & Línea de base (rango) & $\begin{array}{c}\text { Costo/ } \\
\text { beneficio }\end{array}$ \\
\hline Incidencia de dengue ${ }^{a}$ & Intervención & 3,75 & $N A^{b}$ \\
\hline Incidencia de dengue ${ }^{a}$ & No Intervención & $50(0-100)$ & NA \\
\hline Tasa FHD ${ }^{c, d} / F^{e, d}$ & Ambas & $0,1(0-1)$ & NA \\
\hline Internación por FHD f,g & Ambas & 933 & C \\
\hline Medicamentos para FD ${ }^{f, g}$ & Ambas & 7 & $\mathrm{C}$ \\
\hline Medicamentos para $\mathrm{FHD}^{\mathrm{f}, \mathrm{g}}$ & Ambas & 233 & C \\
\hline Reactivos de laboratorio $\mathrm{f}, \mathrm{g}$ & Ambas & 3 & $\mathrm{C}$ \\
\hline Salario médico para atención de FD ${ }^{f, g}$ & Ambas & 33 & $\mathrm{C}$ \\
\hline Combustibles ${ }^{g}$ & Intervención & 32391 & C \\
\hline Insecticida para mosquitos adultos ${ }^{g}$ & Intervención & 51637 & $\mathrm{C}$ \\
\hline Insecticidas para formas inmaduras ${ }^{g}$ & Intervención & 113 & C \\
\hline Material de difusióng & Intervención & 32000 & $\mathrm{C}$ \\
\hline Salario de operarios locales de controlg & Intervención & 59200 & C \\
\hline Salario de técnicos de controlg & Intervención & 120000 & $\mathrm{C}$ \\
\hline \multicolumn{4}{|l|}{ Salario personal de organizaciones } \\
\hline no gubernamentales ${ }^{g}$ & Intervención & 41666 & $\mathrm{C}$ \\
\hline Viajes de epidemiólogosg & Intervención & 4333 & $\mathrm{C}$ \\
\hline Viáticos de epidemiólogosg & Intervención & 30000 & C \\
\hline Ahorro de días laborables por FDe,f,g & Intervención & $219,26(131,56-438,52)$ & B \\
\hline Ahorro de días laborables por FHDe,f,g & Intervención & $613,92(394,67-1315,56)$ & $\mathrm{B}$ \\
\hline \multicolumn{4}{|l|}{ Disponibilidad a pagar por no sufrir } \\
\hline un episodio de dengue $e^{f, g}$ & Intervención & $18,5(12-25)$ & $\mathrm{B}$ \\
\hline \multicolumn{4}{|l|}{ Disponibilidad a pagar por no sufrir } \\
\hline un episodio de dengue hemorrágico & Intervención & 7 075,57 (3 537,78-10 613,35) & B \\
\hline
\end{tabular}

Fuente: elaboración propia.

a Por 1000 habitantes.

b No aplicable.

${ }^{\mathrm{c}}$ Fiebre hemorrágica por dengue.

d Por 100 casos de dengue.

e Fiebre por dengue.

f Por caso.

g Dólares internacionales (I\$).

CUADRO 2. Resultados del análisis de costo-beneficio de dos estrategias, con y sin control vectorial, ciudad de Clorinda, Argentina, enero a abril de 2007

\begin{tabular}{lcccc}
\hline \multicolumn{1}{c}{ Estrategia } & $\begin{array}{c}\text { Costo } \\
(I \$)^{\mathrm{a}}\end{array}$ & $\begin{array}{c}\text { Beneficio } \\
(I \$)^{\mathrm{a}}\end{array}$ & $\begin{array}{c}\text { VPN }^{\mathrm{b}} \\
(\mathrm{I} \$)^{\mathrm{a}}\end{array}$ & $\begin{array}{c}\text { VPN }^{\mathrm{b}} \\
\text { marginal }^{(I \$)^{\mathrm{a}}}\end{array}$ \\
\hline Sin control vectorial & 106724 & 0 & -106724 & NA \\
Con control vectorial & 378372 & 575251 & 196879 & 303602 \\
\hline
\end{tabular}

Fuente: elaboración propia.

a Dólares internacionales.

b Valor presente neto.

morrágico, no demostraron mayor influencia en el resultado.

\section{CONCLUSIONES}

En este estudio se planteó el uso de insecticidas adulticidas en ciclos com- pletos en toda la ciudad, sumado al control de formas inmaduras, como estrategia de prevención ante la inminencia de un brote de dengue por ingreso de personas virémicas desde lugares vecinos y en presencia del vector Aedes aegypti en la ciudad. Esta estrategia se usa en casos de transmisión ya compro- 
FIGURA 2. Análisis de sensibilidad de una vía del parámetro "incidencia de dengue por mil habitantes" y su efecto en el costo con y sin control vectorial, ciudad de Clorinda, Argentina, enero a abril de 2007

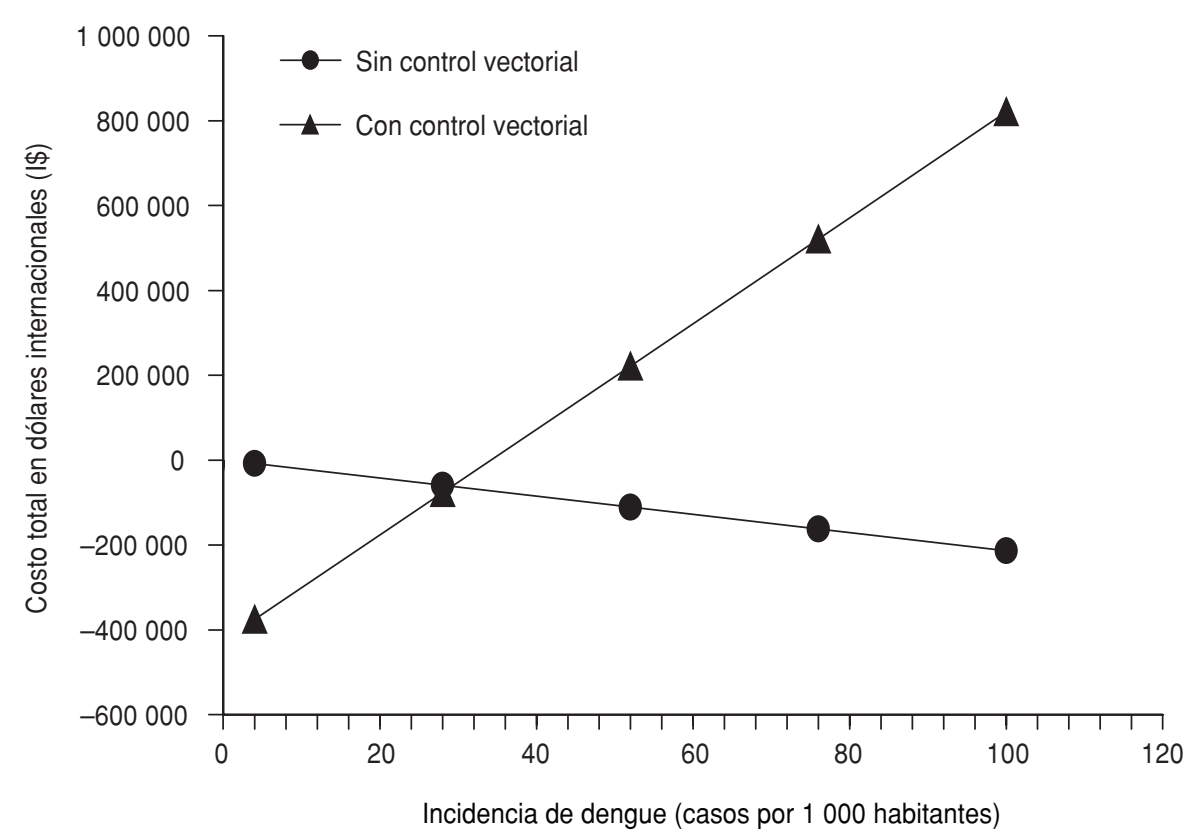

Fuente: elaboración propia.

bada para el corte de la misma. En países como Argentina, este tipo de metodologías cobra importancia debido al corto período con riesgo de transmisión, que generalmente dura entre enero y abril, coincidente con los meses de mayor temperatura y precipitación pluvial. Además, los brotes de dengue en Argentina se asocian con los que ocurren en los países vecinos, lo que se evidencia por los serotipos circulantes en cada región del país, que coinciden con aquellos de las naciones limítrofes. Por lo tanto, el control de mosquitos adultos en ciudades de frontera es una estrategia que podría evitar la aparición de brotes autóctonos. Sin embargo, debido a la baja incidencia del dengue en Argentina, cabría preguntarse si esta estrategia resultaría eficiente. En la literatura existen pocos trabajos publicados sobre costo-efectividad o minimización de costos para dengue, mientras que no se halló ninguna publicación, a conocimiento de los autores, sobre costo-beneficio de estrategias de control de la transmisión del dengue. Cabe destacar que esos trabajos son métodos de evaluación económica más amplios, ya que permiten la comparación directa de los diferentes tipos de variables de resultados (30).

En el presente estudio se determinó que, con una incidencia superior a 29 casos por cada 1000 habitantes (1 363 casos) y más de un caso de dengue hemorrágico, la estrategia de control de mosquitos adultos, incluyendo fumigaciones, es costo-beneficiosa. Esta incidencia es alta si se toma en cuenta el número de casos confirmados en el anterior brote de Clorinda del año 2000. Sin embargo, en esa oportunidad se consideraron exclusivamente los positivos por laboratorio, por lo cual ese número debería ser varias veces superior. Según algunos datos encontrados en la literatura, la incidencia real de dengue podría ser de dos a tres veces superior a la registrada por el sistema de vigilancia de laboratorio (31). En el caso de Argentina, si se toma en cuenta el brote de 2004 en la provincia de Salta, en las localidades de Tartagal, Irigoyen, Orán y Embarcación, los casos registrados por el sistema ascendieron a 1339 (32). Si ocurren brotes en países vecinos es probable entonces que la transmisión su- ceda en las provincias del norte, con una incidencia superior a la observada hasta el momento, debido a la alta cantidad de susceptibles $(33,34)$ y con la posibilidad de aparición de casos de dengue hemorrágico debido a la introducción de más de un serotipo (19). De acuerdo con este escenario, la estrategia de control con adulticidas a través de la fumigación en ciudades fronterizas a áreas con brotes constituiría una alternativa eficiente.

Según lo observado en el presente trabajo sobre los costos que acarrea un brote de dengue, el mayor valor es el generado por el ausentismo laboral de los casos. Otros costos importantes son los salarios de los técnicos y operarios y de los médicos para la atención de pacientes. En el caso de la fiebre por dengue, no se tomó en cuenta la internación porque los casos en general no lo requieren. Sin embargo, la internación por dengue hemorrágico es habitual para control estrecho de la evolución del paciente, y esto significa un gasto importante para el sistema de salud. Además, no todas las localidades con riesgo potencial de transmisión 
poseen salas de cuidados intensivos, por lo que habría que considerar también el traslado del paciente hacia otras ciudades con hospitales de mayor complejidad, y el riesgo que ello conlleva. Por otro lado, el presente estudio no tomó en cuenta tampoco el impacto ambiental o social de las fumigaciones para el control de adultos, ni tampoco el riesgo de desarrollo de resistencia a insecticidas, debido a que se trató de una experiencia puntual ante determinados escenarios de riesgo y no una estrategia de aplicación sistemática.

En definitiva, los resultados de este estudio sugieren que, bajo el escenario propuesto, la estrategia de fumigación para control de insectos adultos debe ser evaluada cuidadosamente y sería costo-beneficiosa junto con acciones de control de formas inmaduras en países no endémicos como Argentina ante el riesgo inminente de transmisión, para evitar el comienzo de la misma.

\section{REFERENCIAS}

1. Teixeira Mda G, Costa Mda C, Barreto ML, Mota E. Dengue and dengue hemorrhagic fever epidemics in Brazil: what research is needed based on trends, surveillance, and control experiences? Cad Saude Publica. 2005; 21(5):1307-15.

2. World Health Organization. Dengue haemorrhagic fever. Diagnosis, treatment, prevention and control. $2^{a}$ ed. Geneva: WHO; 1997.

3. San Martín JL, Brathwaite-Dick O. La estrategia de gestión integrada para la prevención y el control del dengue en la Región de las Américas. Rev Panam Salud Publica. 2007; 21(1):55-63.

4. Hombach J. Vaccines against dengue: a review of current candidate vaccines at advanced development stages. Rev Panam Salud Publica. 2007;21(4):254-60.

5. Ashford DA, Savage HM, Hajjeh RA, McReady J, Bartholomew DM, Spiegel RA, et al. Outbreak of dengue fever in Palau, Western Pacific: risk factors for infection. Am J Trop Med Hyg. 2003;69(2):135-40.

6. Aviles G, Rangeon G, Vorndam V, Briones A, Baroni $P$, Enria D, et al. Dengue reemergence in Argentina. Emerg Infect Dis. 1999; 5(4): $575-8$.

7. Torres JR, Castro J. The health and economic impact of dengue in Latin America. Cad Saude Publica. 2007; 23 Suppl 1:S23-31.

8. Shepard DS, Suaya JA, Halstead SB, Nathan MB, Gubler DJ, Mahoney RT, et al. Costeffectiveness of a pediatric dengue vaccine. Vaccine. 2004;12; 22(9-10):1275-80.

9. Meltzer MI, Rigau-Perez JG, Clark GG, Reiter $\mathrm{P}$, Gubler DJ. Using disability-adjusted life years to assess the economic impact of dengue in Puerto Rico: 1984-1994. Am J Trop Med Hyg. 1998;59(2):265-71.

10. Cho-Min-Naing. Assessment of dengue hemorrhagic fever in Myanmar. Southeast Asian J Trop Med Public Health. 2000;31(4):636-41.

11. Clark DV, Mammen MP Jr, Nisalak A, Puthimethee V, Endy TP. Economic impact of dengue fever/dengue hemorrhagic fever in Thailand at the family and population levels. Am J Trop Med Hyg. 2005;72(6):786-91.

12. Anderson KB, Chunsuttiwat $S$, Nisalak A, Mammen MP, Libraty DH, Rothman AL, et al. Burden of symptomatic dengue infection in children at primary school in Thailand: a prospective study. Lancet. 2007;369(9571): 1452-9.

13. McConnell KJ, Gubler DJ. Guidelines on the cost-effectiveness of larval control programs to reduce dengue transmission in Puerto Rico. Rev Panam Salud Publica. 2003;14(1):9-16.

14. Toledo ME, Vanlerberghe V, Baly A, Ceballos E, Valdes L, Searret M, et al. Towards active community participation in dengue vector control: results from action research in Santiago de Cuba, Cuba. Trans R Soc Trop Med Hyg. 2007;101(1):56-63.

15. Cabrera A. Regiones fitogeográficas argentinas. Enciclopedia Argentina de Agricultura y Jardinería. Tomo II. $2^{\mathrm{a}}$ ed. Buenos Aires: Editorial ACME; 1976.

16. Bono J, Parmuchi MG, Gasparri I, Montenegro C, Strada M, Manghi E. Mapa forestal Provincia de Formosa. Actualización año 2002. [Sitio en Internet] Hallado en: http:// www2.medioambiente.gov.ar/documentos/ bosques/umsef/cartografia/2002_formosa. pdf. Acceso el 7 julio 2007.

17. Instituto Nacional de Estadísticas y Censos de Argentina. Resultados Provinciales del Censo 2001. [Sitio en Internet] Hallado en: http://www.indec.mecon.gov.ar/webcenso/ provincias_2/provincias.asp. Acceso el 12 mayo 2007.

18. Boletín Epidemiológico Nacional. República Argentina: Ministerio de Salud y Ambiente de la Nación; 2003.

19. Aviles G, Paz MV, Rangeon G, Ranaivoarisoa MY, Verzeri N, Roginski S, et al. Laboratory surveillance of dengue in Argentina, 19952001. Emerg Infect Dis. 2003; 9(6):738-42.

20. Masuh H, De Licastro SA, Lopez PA, Vega C, Zerba E. Field evaluation of a smoke-generating formulation containing beta-cypermethrin against the dengue vector in Argentina. J Am Mosq Control Assoc. 2003; 19(1):53-7.

21. Ministerio de Salud de la Nación. Argentina (2007). Actualización de la situación de dengue. Argentina - 1 de junio de 2007. [Sitio en Internet] Hallado en: http://www.msal gov.ar/htm/Site/actualizacion_limitrofes_ dengue_y_argentina_01_de_junio_de_2007. xls. Acceso el 8 junio 2007.

22. Pan American Health Organization, 2006. Number of reported cases of dengue \& dengue hemorrhagic fever, region of the
Americas by country and subregion, $\mathrm{PAHO}$, Washington. [Sitio en Internet] Hallado en: http://www.paho.org/Spanish/AD/DPC/ $\mathrm{CD} /$ dengue.htm. Acceso el 14 junio 2007.

23. Ministerio de Trabajo, Empleo y Seguridad Social de Argentina. Ingresos, salarios y costo laboral. Ingreso medio de los ocupados plenos según variables seleccionadas. Subsecretaría de Programación Técnica y Estudios Laborales - Dirección General de Estadísticas y Estudios Laborales, en base a EPH (INDEC); 2006. [Sitio en Internet] Hallado en: http://www.trabajo.gov.ar/left/estadisticas/ bel/downloads/xls/2121.xls. Acceso el 10 de junio de 2008.

24. Drummond M, O'Brien B, Stoddart G, Torrance G. Métodos para la evaluación económica de los programas de asistencia sanitaria. $2^{a}$ ed. Madrid: Ediciones Díaz de Santos S.A.; 2001.

25. Lee PY, Matchar DB, Clements DA, Huber J Hamilton JD, Peterson ED. Economic analysis of influenza vaccination and antiviral treatment for healthy working adults. Ann Intern Med. 2002; 137(4):225-31.

26. Prosser LA, Bridges CB, Uyeki TM, Rêgo VH, Ray GT, Meltzer MI, et al. Values for preventing influenza-related morbidity and vaccine adverse events in children. Health Qual Life Outcomes. 2005; 3:18.

27. Yasunaga $H$, I de $H$, Imamura $T$, Ohe K. Willingness to pay for health care services in common cold, retinal detachment, and myocardiac infarction: an internet survey in Japan. BMC Health Serv Res. 2006; 6: 12.

28. Yasunaga $H$, Ide $H$, Imamura $T$, Ohe $K$. Analysis of factors affecting willingness to pay for cardiovascular disease-related medical services. Int Heart J. 2006;47(2):273-86.

29. The World Bank Group. 2006 World Development Indicators. Exchange rates and prices. [Sitio en Internet] Hallado en: http://devdata. worldbank.org/wdi2006/contents/Table4_ 14.htm. Acceso el 8 junio 2007.

30. Haddix A, Teutsch S, Corso P. Prevention effectiveness: A guide to decision analysis and economic evaluation. $2^{\mathrm{a}}$ ed. Oxford: Oxford University Press, Inc; 2003.

31. Dechant EJ, Rigau-Perez JG. Hospitalizations for suspected dengue in Puerto Rico, 19911995: estimation by capture-recapture meth- 
ods. The Puerto Rico Association of Epidemiologists. Am J Trop Med Hyg. 1999;61(4): 574-8.

32. Boletín Epidemiológico Nacional 2004/2005. Ministerio de Salud, Presidencia de la Nación, República Argentina; 2006.

33. Gorodner JO, Seijo A, Payes MF, Robles B, Basualdo $\mathrm{C}$, Pacce $\mathrm{M}$, et al. Circulación de virus dengue en el nordeste argentino. Comunicaciones Científicas y Tecnológicas de la Uni- versidad Nacional del Nordeste, Corrientes. [Sitio en Internet] Hallado en: http://www. unne.edu.ar/Web/cyt/cyt/2000/3_medicas/ m_pdf/m_019.pdf. Acceso el 25 junio 2007.

34. Gorodner JO, Seijo A, Pacce M, Bottinelli O, Merino DE, Lazarte M, et al. Circulación de virus Dengue en la Provincia de CorrientesArgentina. Comunicaciones Científicas y Tecnológicas de las Universidad Nacional del Nordeste, Corrientes. [Sitio en Internet] Ha- llado en: http://www.unne.edu.ar/Web/cyt/ cyt/2001/3-Medicas/M-060.pdf Acceso el 25 junio 2007.

Manuscrito recibido el 14 de septiembre de 2007. Aceptado para publicación, tras revisión, el 29 de abril de 2008.

ABSTRACT Objectives. To evaluate the cost-benefit of an intervention utilizing fumigation against adult mosquitoes along with actions to control immature Aedes aegypti to prevent transmission in a high-risk area.

\section{Cost-benefit analysis of vector control in areas of potential dengue transmission}

Methods. A cost-benefit analysis was performed from the social perspective. A program to control both adult mosquitoes and immature forms was compared to a nonintervention hypothesis, from January to April 2007, in the city of Clorinda, Argentina. Direct costs, as well as indirect and intangible benefits, were taken into account and standardized in international dollars (I\$) using purchasing power parity. An incidence rate of 50 cases of dengue per 1000 inhabitants was used in the non-intervention hypothesis.

Results. From January to April 2007 there were 176 cases of dengue in Clorinda, but only five were autochthonous. The net present value was I\$ 196879 with the control strategy, whereas the non-intervention was calculated to be I\$ -106724 , indicating a savings of I\$ 303602 (I\$ 6.46 per inhabitant) for the intervention. The sensitivity analysis showed that, with more than 1363 cases of dengue (total incidence rate of 29 per 1000 inhabitants) and one case of dengue hemorrhagic fever, there is a cost-benefit to intervention.

Conclusions. The results suggest that vector control, including fumigation of adult mosquitoes, should be considered in high-risk areas as a cost-effective option for nonendemic countries.

Key words Cost-benefit analysis, dengue, vector control, Aedes, Argentina. 